Sandra Fancello, André Mary (éd.), Chrétiens africains en Europe, Prophétisme, pentecôtisme et politique des nations

Paris, Karthala, coll. « Religions contemporaines », 2010, 420 p.

\title{
Sébastien Fath
}

\section{(2) OpenEdition \\ Journals}

\section{Édition électronique}

URL : http://journals.openedition.org/assr/23954

DOI : $10.4000 /$ assr.23954

ISSN : $1777-5825$

\section{Éditeur}

Éditions de l'EHESS

Édition imprimée

Date de publication : 30 décembre 2012

ISSN : 0335-5985

\section{Référence électronique}

Sébastien Fath, « Sandra Fancello, André Mary (éd.), Chrétiens africains en Europe, Prophétisme, pentecôtisme et politique des nations ", Archives de sciences sociales des religions [En ligne], 160 | octobre-décembre 2012, mis en ligne le 28 janvier 2014, consulté le 21 septembre 2020. URL : http:// journals.openedition.org/assr/23954; DOI : https://doi.org/10.4000/assr.23954 


\section{Sandra Fancello, André Mary (éd.), Chrétiens africains en Europe, Prophétisme, pentecôtisme et politique des nations}

Paris, Karthala, coll. « Religions contemporaines », 2010, 420 p.

Sébastien Fath

\section{RÉFÉRENCE}

Sandra Fancello, André Mary (éd.), Chrétiens africains en Europe, Prophétisme, pentecôtisme et politique des nations, Paris, Karthala, coll. « Religions contemporaines ", 2010, $420 \mathrm{p}$. 
1 La somme dirigée par Sandra Fancello et André Mary prolonge, complète et amplifie un remarquable numéro thématique des Archives de Sciences Sociales des Religions intitulé "Christianismes du Sud à l'épreuve de l'Europe » (coordonné par Sandra Fancello et André Mary, n¹43, juillet-septembre 2008). Publiée aux éditions Khartala, elle se subdivise en trois parties : «Prophètes et nations » (I), "Individus et communautés» (II) et « Nouveaux espaces » (III). L'ambition est vaste, adossée à un évident souci

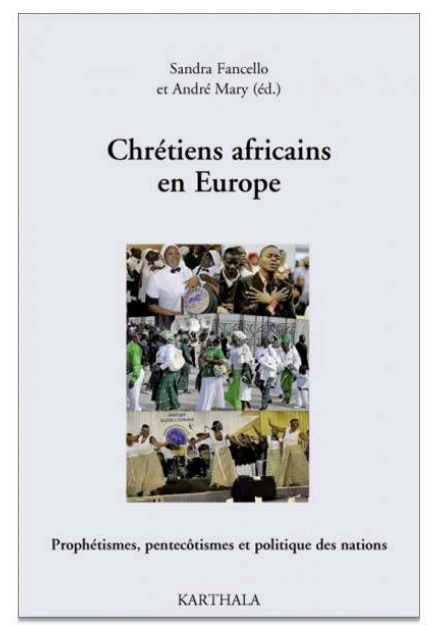
d'étendre le périmètre scientifique ouvert par ces nouveaux objets d'étude, quitte à prendre des risques: "cette rencontre étonnante sur le terrain du langage de la force spirituelle entre le fondamentalisme évangélique de l'Esprit de puissance et la sacralité territoriale des charismes prophétiques africains bouscule nos évidences ontologiques régionales et notre rationalité éthique » (Fancello et Mary, p. 36).

2 La première partie est composée de cinq contributions. Dans une étude originale rédigée en anglais, Ramon Sarro (Institut des Sciences Sociales de Lisbonne) et Anne Mélice (Université de Liège) commencent par analyser la dialectique centre-périphérie au sein de l'Église kimbanguiste, Église congolaise issue de l'activité prophétique de Simon Kimbangu (1887-1951). Les auteurs rappellent l'originalité du kimbanguisme par rapport aux pentecôtismes et néo-pentecôtismes, sur la base d'une doctrine du SaintEsprit fondée sur l'identification avec le prophète Simon Kimbangu. Ils mettent en lumière le lien privilégié, véritable « cordon ombilical » (p. 60) qui relie kimbanguisme congolais et Portugal au travers de l'histoire de Kimpa Vita, prophétesse congolaise condamnée à mort en 1706, deux siècles après les débuts de l'aventure coloniale portugaise au Congo.

3 Appuyée sur une abondante documentation (où manque néanmoins la mention des travaux d'Aurélien Mokoko Gampiot), cette étude (p. 43-67) est suivie par une seconde monographie originale en anglais, consacrée cette fois à l'Église tokoïste d'Angola. Signée par Ruy Liera Blanes (Institut des Sciences Sociales de Lisbonne), elle jette une lumière originale sur une Église jusqu'alors assez peu connue en dehors d'un cercle restreint de spécialistes, «l'Église tokoïste », mouvement réformiste prophétique, né en Angola sous l'impulsion de Simon Toko (1918-1984). Cette Église, qui constituerait aujourd'hui le second acteur religieux le plus important d'Angola après l'Église catholique, s'appuie sur un réseau diasporique dense et une dynamique charismatique complexe, bousculée par la mort du fondateur en 1984. Après une période de turbulence, la personnification de l'esprit du défunt fondateur en Alfonso Nunes, en l'an 2000, marque une relance du mouvement sur la base d'une globalisation du charisme teintée de messianisme africain et de culture pentecôtiste globalisée (p. 69-91).

4 Bernard Coyault recentre ensuite l'analyse sur trois itinéraires individuels, qui emblématisent une forme religieuse qui ne s'est pas laissée dissoudre par la dynamique pentecôtiste transnationale. Cette forme est le Nsikumusu, courant prophétique africain 
qui marque l'aire culturelle Kongo, et dans lequel s'inscrivent trois prophétesses de l'Église Evangélique du Congo résidant en France. Au travers d'Elise, de Rose et de Sophie, l'auteur élucide la tension entre fidélité aux formes prophétiques héritées et adaptation à une clientèle diasporique et française aux demandes spécifiques (p. 93 à 125). Il souligne l'opportunité de réfléchir à un "scénario alternatif, non pas seulement» marqué par «la pentecôtisation des prophétismes» (une tendance majoritaire), mais aussi par «la 'prophétisation' - ou l'indigénisation - des pentecôtismes » (p. 121).

Dans sa contribution intitulée "Christianisme prophétique et Nations Célestes », André Mary développe ensuite, des pages 127 à 153, une analyse des formes du «combat spirituel pour la purification du monde » dans l'Église du Christianisme Céleste, fondée au Bénin en 1947. Ces enjeux s'inscrivent dans un contexte très particulier : celui de la mort, le 13 avril 2010 à Porto Novo, de Benoît Agbaossi, considéré comme le chef suprême de l'Église après la disparition en 1985 du fondateur, le prophète Samuel Biélou Oschoffa. Au travers d'une description anthropologique très fine, l'auteur traite des querelles de succession et de légitimité, et pointe notamment les convergences entre les pratiques de délivrance du Christianisme céleste et les "pratiques charismatiques ou pentecôtistes de délivrance qui s'offrent aujourd'hui aux Antilles, en Afrique et en Europe, sur le même marché de la guérison divine» (p. 138). Il relève cependant aussi des "différences significatives", à la fois en matière rituelle et en matière symbolique. Ainsi, «dans la prière de combat des Célestes, c'est la puissance des anges et particulièrement de l'Archange Saint Michel qui est censée intervenir », au contraire d'un habitus pentecôtiste centré sur la seule puissance de l'Esprit Saint.

6 Damien Mottier clôt la première partie thématique de l'ouvrage par une synthèse intitulée "Réveils prophétiques et entreprenariat charismatique», au travers de l'étude du «creuset congolais » et du « prophétisme ivoirien en France» (p. 155 à 178). Cette contribution majeure à la sociohistoire des scissiparités protestantes franciliennes des trente dernières années éclaire les logiques de redéfinition du charisme prophétique au travers d'études de cas emblématiques (Mathieu Kayeye, Kacou Séverin, Selvaraj Rajiah), sur fond de dynamiques de champ visant à mobiliser les nouvelles clientèles afro-évangéliques au nom de la « reconquête spirituelle d'une Europe matérialiste et moralement en danger » (p. 177). On pardonnera d'autant plus à cette étude très maîtrisée un renvoi oublié (citation de Fath, 2005, à la page 176, qu'on ne retrouve pas en bibliographie) qu'elle articule avec beaucoup de rigueur réélaboration critique d'abondants matériaux empiriques, et éclairage théorique multifocal.

7 La seconde grande partie du livre intègre quatre contributions. Elle est intitulée «Individus et communautés». Elle s'ouvre sur la contribution d'Alessandro Gusman (anthropologue à l'Université de Turin), intitulée «J'étais seul quand j'ai rencontré Jésus ». Au travers d'une étude sur les conversions au pentecôtisme à Turin dans le contexte migratoire, on découvre, entre les pages 181 et 205, une des premières analyses de fond consacrées à l'impact des « vagues massives d'immigration » (p. 181) sur le paysage religieux italien. Dans la seule ville de Turin, début 2010, on recensait ainsi 15 Églises africaines.

8 A l'aide d'une approche typologique, l'auteur éclaire la fonction sociale essentielle jouée par les Églises, soulignant combien «il n'est pas possible d'ignorer le caractère pragmatique de la conversion pentecôtiste » (p. 194). 
9 Le caractère socialement utile de la conversion est cependant loin de résumer la palette, complexe, des motivations des acteurs, comme le souligne ensuite Sandra Fancello (CNRS) dans sa contribution, intitulée "'Afrique élève l'Europe': pentecôtisme, afrocentrisme et démocratie » (p. 207 à 241). L'auteure s'appuie sur un riche terrain anthropologique, celui des conférences " Afrique élève l'Europe ", tenues chaque été à Bruxelles depuis 2004, pour ausculter l'enjeu de la "mission inversée ", qu'elle résume ainsi : «ramener l'Évangile aux Européens qui s'en sont détournés ». Sandra Fancello précise d'emblée que ce discours «relève plus souvent d'une rhétorique que d'une stratégie réelle", mais qu'il n'en contribue pas moins à " précipiter la rencontre missionnaire entre chrétiens européens et africains en Europe, confrontés à un choc culturel réciproque» (p.207). Fondée sur une rhétorique conservatrice, conquérante, performative, où les "nationalités politiques » se superposent aux « nations bibliques » avec leurs «territoires spirituels» (p. 234), cette « rencontre » révèle bien des ambiguïtés, analysées avec précision par l'auteure. Un exemple parmi d'autre? Ce désir de " réinvestissement dans le pays d'origine ", finalement prioritaire sur "l'intégration dans les sociétés européennes " (p. 235). En dehors d'une erreur de détail (Obama, présenté comme un "protestant évangélique », ce qu'il n'est nullement, p. 214), cette étude témoigne d'une impressionnante capacité à contextualiser, à partir d'un objet particulièrement complexe (Églises africaines de multiples origines et sensibilités).

10 La focale se resserre ensuite sur un objet beaucoup plus ciblé, celui d'une Église gabonaise en France, étudiée par Maixent Mebiame Zomo, de l'Université Ombar Bongo à Libreville (p. 243 à 272). Au travers de trajectoires individuelles entre le Gabon, Paris et Amiens (Picardie), l'auteur lève le voile sur les ambitions missionnaires de la Communauté Béthanie qui fonde « son expansion sur la migration étudiante gabonaise et quelques professionnels de passage»(p.270). Cette stratégie s'appuie sur un processus de recommunautarisation des migrants gabonais, sur le modèle déjà observé par Sandra Fancello en contexte ghanéen, mais aussi sur une dynamique prosélyte en direction du pays d'accueil, doublée d'un accent sur l'intégration. Sabine Jaggi, formée à l'Université de Berne et à l'EHESS, ferme le ban de la seconde partie de l'ouvrage avec une monographie éclairante consacrée à La Vigne de Berne, une Église africaine dans la Suisse d'aujourd'hui confrontée aux enjeux de l'insertion dans le tissu ecclésial existant (p. 273 à 298). Très bien contextualisée dans le cadre général de la diversité cantonale et religieuse suisse, cette étude décrit les modalités du processus au terme duquel La Vigne de Berne est parvenue à entretenir de bonnes relations avec les milieux ecclésiastiques et les acteurs politiques du canton de Berne. Intervenue sur fond de durcissement des politiques migratoires, cette évolution n'élimine pas les tensions, comme l'illustre la conclusion de l'étude: à la question, posée par « Lionel, membre de la Vigne de Berne ", de savoir si les fidèles sont d'abord frères en Christ ou patriotes, Sabine Jaggi fait observer: "L'auditoire se renferma dans un silence consterné " (p. 296).

11 Déjà très riche, l'ouvrage est complété par une troisième et dernière partie qui rassemble quatre textes consacrés aux dynamiques spatiales («Nouveaux espaces»). Frédéric Dejean y mobilise ses talents de géographe pour analyser la " précarité spatiale des Églises africaines en Seine-Saint-Denis et sur l'île de Montréal » (p. 301 à 325). Dans une approche originale et maîtrisée, l'auteur (actuellement post-doc à l'Université de Montréal) s'intéresse à la «mise en visibilité » des Églises de migrants 
au travers d'une comparaison transatlantique fondée sur deux métropoles marquée chacune par l'immigration. Observant les difficultés d'accès à l'espace en contexte parisien, liées à des contraintes qu'on ne retrouve pas à Montréal, ville nordaméricaine plus récente où la densité du bâti est bien plus faible, l'auteur met en avant le caractère crucial de l'accessibilité, et le grand degré d'adaptation des Églises africaines, qui parviennent à subvertir «les contraintes posées par la ville postindustrielle pour les intégrer pleinement dans leur oeuvre missionnaire » (p. 322).

Maskens (Université Libre de Bruxelles) développe ensuite une analyse des logiques prosélytes mises en oeuvre dans une assemblée de migrants africains hispanophones à Bruxelles, le Centro Emmanuel («Semer des 'graines divines' dans le coeur des prostituées à Bruxelles», p. 327-349). La seconde partie de l'article s'inscrit dans une démarche monographique classique, étayée par d'abondants matériaux primaires (4 pages et demi de transcription du journal de terrain, des pages 337 à 341 !). On y découvre les logiques intentionnelles des acteurs, confrontés à un public difficile d'accès, aux aguets et peu coopératif, et les logiques objectives d'une entreprise prosélyte qui échoue à rallier de nouveaux fidèles, mais qui atteint « d'autres objectifs propres à la constitution de l'identité religieuse de l'individu et de la construction de la collectivité charismatique » (p. 345). Cette étude monographique rondement menée est complétée, dans les cinq premières pages de l'article, par une entrée en matière plus théorique, particulièrement claire et maîtrisée, qui permet d'expliquer en quoi l'entreprise missionnaire n'est pas seulement « un acte effectué dans l'unique but de convertir d'autres personnes, mais c'est aussi et avant tout un mode d'action rhétorique qui crée ou recrée le soi pentecôtiste»(p.332), dans le registre de l'hétérotopie (p. 343-344).

Quittant la Belgique pour la Suisse, on découvre, avec Jeanne Rey (Institut des Hautes Etudes Internationales et du Développement à Genève), les problématiques spécifiques liées à la forme de "l'Église internationale ", conçue avant tout pour une clientèle d'expatriés de diverses nationalités. Il ne s'agit pas ici nécessairement d'une Église de migrants, en tout cas au sens habituel du terme. Jeanne Rey inscrit cependant son étude dans le contexte plus large des "Églises évangéliques étrangères ", que le Centre intercantonal d'Information sur les Croyances évaluait à plus de soixante en 2009. Mais le terreau de recrutement porte ici sur «les organisations internationales et l'Organisation des Nations Unies ", qui attirent à Genève "des fonctionnaires et diplomates en provenance de nombreux Etats » (p. 355). On s'inscrit donc dans un imaginaire international, lequel se déploie « dans un chronotope particulier, celui des prophéties millénaristes » (p. 359). L'étude fine des rituels mis en oeuvre révèle une oscillation ambigüe entre registre biblique et connotation géopolitique, dans la mise en musique d'une "conquête des nations" prise très au sérieux par la communauté. L'activité rituelle, souligne l'auteure, constitue précisément le moyen au travers duquel les fidèles se réapproprient une capacité d'action en s'associant à l'agir de Dieu, qui dans le mode de pensée eschatologique est seul agent direct de la construction millénariste.

14 Enfin, la dernière contribution, qui clôture le volume, est signée par l'historien brésilien Edio Soares. Elle porte aussi sur le terrain hélvétique et genevois, au travers de l'implantation de l'Église Universelle du Royaume de Dieu dans la capitale du canton de Vaud, sur la base d'une communauté de 250 membres, constituée d'abord de migrants brésiliens et portuguais, puis enrichie de migrants africains (p. 381 à 407). La 
méthodologie de l'auteur est celle de l'anthropologue, mais sans négliger une robuste contextualisation historique, qui situe l'arrivée en Suisse de l'EURD en 1993. Les spécificités doctrinales de cette Église, déjà bien connues grâce aux travaux pionniers de Marion Aubrée (que l'auteur cite à juste titre) sont définies autour d'un rapport transactionnel et contractualisé avec la divinité trine, fondé sur l'efficacité de la parole performative: " au lieu d'attendre les grâces que Dieu veut bien lui octroyer, (le croyant) doit 'exiger' celles-ci non plus comme une récompense pour une vie ascétique mais comme une restitution contractuelle, tant pour la foi qu'il professe que pour les dons en argent qu'il fait à l'Église universelle " (p. 393). L'étude des "manières de faire » de l'Église met aussi l'accent sur la notion brésilienne de jeitinho (arrangement), qui souligne qu'au-delà des formes autoritaires prises par cette Église néo-pentecôtiste très hiérarchique, l'EURD témoigne d'une grande souplesse d'adaptation, au service d'une clientèle qui demeure avant tout constituée de migrants soucieux d'ascension sociale.

Le survol trop rapide des monographies présentées successivement dans le volume dirigé par Sandra Fancello et André Mary ne saurait constituer une fin en soi, bien qu'à lui seul, ce vaste corpus d'études, bien thématisé, représente un jalon scientifique majeur. Il faut en effet souligner, pour parachever le tableau d'ensemble, l'extrême fécondité théorique et méthodologique de l'ample introduction (p. 11 à 40) signée par les deux maîtres d'oeuvre du livre. Cette introduction magistrale, à elle seule, suffit à justifier de s'approprier ce livre, tant elle permet au chercheur d'affûter ses outils, d'améliorer sa visibilité du champ et d'éviter les chausse-trappes. Non contents de contextualiser et d'historiciser le phénomène des Églises africaines en Europe, Sandra Fancello et André Mary s'attardent utilement sur les enjeux taxinomiques (quelle terminologie descriptive étudier ?), problématisent les conflits d'authenticité posés par l'offre religieuse alternative proposée par ces nouvelles Églises, et mettent en avant (p.18) un paradigme jugé désormais dominant (sans doute à raison) : celui des PCC (Pentecostal and Charismatic Churches), cadre dans lequel s'inscrivent toutes les Églises étudiées dans la suite du volume. S'appuyant aussi sur la notion heuristiquement féconde de «territoire circulatoire » popularisée par Alain Tarrius, les auteurs font justement valoir le fait que l'idée « d'une 'communauté diasporique', qui relie ceux qui sont partis et ceux qui sont restés, subvertit complètement la linéarité des parcours migrants qui primaient dans les travaux plus anciens » ( $p$.22). Les auteurs n'oublient pas de rappeler, chemin faisant, que si les «nations africaines ont du mal à rompre avec les 'ethno-nations' qui ont marqué leur histoire et hantent leur présent ", "que dirait-on si les appellations nationales européennes étaient traitées comme des marqueurs ethniques?» (p. 23).

S'appuyant sur un extrait éclairant et très bienvenu de Jean Baubérot sur la « négritude protestante » (tiré de Le protestantisme doit-il mourir ? 1988, p. 133-135, mis en exergue aux pages 9 et 10 de l'ouvrage), Sandra Fancello et André Mary insistent aussi sur un point fondamental pour qui veut étudier ces milieux religieux : attention aux « pièges de l'effacement généreux des différences ». (p. 25). Le chercheur a besoin, pour affiner son analyse, de connaitre l'origine des fidèles qu'il étudie, leur statut générationnel, leur étiquette ethnique, sans être dupe pour autant des risques d'essentialisme. Ce point précisé, les auteurs ne s'arrêtent pas en si bon chemin, toutes les thématiques sensibles à l'intérieur du champ d'étude sont passées en revue et problématisées au fil de l'introduction: mission inversée, politique des nations, spiritual mapping... Les auteurs prennent soin, au passage, d'inviter le chercheur en sciences sociales à 
redoubler de prudence face au risque de "pentecôtisation des paradigmes » (p. 30-31) qui menace notamment d'absorber sans distance critique suffisante certains matériaux discursifs propres à la rhétorique conquérante des born again, dont Sandra Fancello et André Mary rappellent qu'elle n'est pas forcément aussi universaliste et cosmopolite qu'elle en a l'air, notamment à cause de sa dénonciation très clivante des outsiders (non croyants, croyants d'autres religions, etc.).

On pourra certes réagir, ça et là, devant certaines généralisations un peu hâtives. "L'absence de conversion effective des 'Blancs'», mentionnée p. 28, pourrait par exemple être légèrement nuancée à partir de certains « terrains » extérieurs à ceux que l'on a sélectionnés ici. Affirmer par ailleurs que "les politiques de reconquête spirituelle des Églises évangéliques sont inséparables d'une politique des Nations » (p.32) est beaucoup trop simpliste. Ce registre mobilisateur est certes devenu très clairement dominant aujourd'hui au sein des PCC (Pentecostal and Charismatic Churches), mais il n'en est pas de même dans les rangs de la mouvance évangélique de type piétiste et orthodoxe (assemblées de frères, méthodisme, baptisme, Églises libres...), où la thématique des "nations » est soit absente, soit parcimonieusement utilisée, au nom d'une méfiance principielle pour l'imaginaire de «l'Église nationale » ou «l'Eglise de multitude », réputé proche du référentiel vétérotestamentaire, dont l'évangélisme, fondé sur la conversion individuelle, s'est fortement démarqué. D'une façon générale, le spécialiste des protestantismes trouvera quelques imprécisions ou arrêtes qui gagneraient à être rabotées ou affinées. Mais, rétorquera-t-on, les spécialistes du protestantisme (et du christianisme en général) seraient bien inspirés, à l'inverse, de se pencher de très près sur la monumentale entreprise comparatiste poursuivie dans cet ouvrage par Sandra Fancello, André Mary et leurs collègues. Comment continuer à étudier le protestantisme contemporain, même lorsqu'on n'est ni anthropologue ni sociologue, en ignorant le champ de recompositions des Églises africaines?

$\mathrm{Au}$ terme de la lecture de ce livre dense et passionnant, l'énorme apport empirique, méthodologique et théorique rassemblé ici force le respect. Depuis le beau travail de défrichage synthétique de Gerrie Ter Haar (Halfway to Paradise. African Christians in Europe, Cardiff Academic Press, 1998), on attendait que l'essai soit transformé... Sandra Fancello, André Mary et leur équipe ont fait mieux que cela. Cet ouvrage majeur et programmatique ne doit pas être réservé au cercle des africanistes. Il est indispensable aux chercheuses et chercheurs qui se penchent sur les recompositions religieuses contemporaines. Par son ambition théorique maîtrisée, sa cohérence polyphonique et sa richesse factuelle, il peut être rangé sans hésiter parmi les meilleures productions en sciences sociales des religions des trente dernières années. 